OPEN ACCESS

Edited by:

George Tsiamis,

University of Patras, Greece

Reviewed by:

Alba Rodriguez Nogales,

University of Granada, Spain

Ming Li,

Dalian Medical University, China

*Correspondence:

Ke Yuan

yuan_ke001@126.com

Specialty section:

This article was submitted to

Systems Microbiology,

a section of the journal

Frontiers in Microbiology

Received: 26 February 2019

Accepted: 05 June 2019

Published: 25 June 2019

Citation:

Cui H-X, Zhang L-S, Luo Y, Yuan K, Huang Z-Y and Guo Y (2019) A Purified Anthraquinone-Glycoside

Preparation From Rhubarb Ameliorates Type 2 Diabetes Mellitus by Modulating the Gut Microbiota

and Reducing Inflammation.

Front. Microbiol. 10:1423.

doi: 10.3389/fmicb.2019.01423

\section{A Purified Anthraquinone-Glycoside Preparation From Rhubarb Ameliorates Type 2 Diabetes Mellitus by Modulating the Gut Microbiota and Reducing Inflammation}

\author{
Hong-Xin Cui ${ }^{1,2}$, Ling-Shuai Zhang ${ }^{1}$, Yang Luo ${ }^{3}$, Ke Yuan ${ }^{3 *}$, Zhi-Yong Huang ${ }^{4}$ and \\ Ying Guo ${ }^{4}$
}

'College of Pharmacy, Henan University of Chinese Medicine, Zhengzhou, China, ${ }^{2}$ Collaborative Innovation Center for Respiratory Disease Diagnosis and Treatment \& Chinese Medicine Development of Henan Province, Zhengzhou, China, ${ }^{3}$ Jiyang College of Zhejiang Agriculture and Forestry University, Zhuji, China, ${ }^{4}$ Zhejiang Chinese Medical University, Hangzhou, China

Rheum palmatum $\mathrm{L}$. is widely used in traditional Chinese medicine for the treatment of constipation. Here, the therapeutic effects and underlying mechanisms of purified anthraquinone-glycoside preparation from rhubarb (RAGP) on the type 2 diabetes mellitus (T2DM) rats were investigated. After 6 weeks of metformin and RAGP treatment, the weight returned to normal. Fasting blood glucose (FBG), glycated serum protein (GSP), insulin concentration and HOMA-IR index had significantly decreased, and glucagon-like peptide-1 (GLP-1) concentrations had increased. Histological abnormalities in the pancreas and ileum had improved. These effects were associated with enhanced intestinal integrity, thereby reducing the absorption of lipopolysaccharide (LPS) and inflammation. To investigate whether RAGP ameliorated insulin resistance via effects on the gut microbiota, we performed $16 \mathrm{~s}$ rDNA sequencing of ileal gut contents. This showed an amelioration of gut dysbiosis, with greater abundance of probiotic Lactobacillus and short-chain fatty acid-producing bacteria, and lower abundance of the Lachnospiraceae NK4A136 group and LPS-producing Desulfovibrio. The mechanism of the hypoglycemic effect of RAGP involves regulation of the gut microbiota, activation of the GLP-1/CAMP pathway to ameliorate insulin resistance. Thus, this study provides a theoretical basis for the use of RAGP to treat T2DM, and it may be a novel approach to restore the gut microbiota.

Keywords: anthraquinone-glucoside, diabetes, gut microbiota, intestinal integrity, inflammation

Abbreviations: AEG, aloe-emodin-8-O- $\beta$-D-glycoside; CPG, chrysophanol-8-O- $\beta$-D-glycoside; EMG, emodin-3-O$\beta$-glycoside; FBG, fasting blood glucose; GLP-1, glucagon-like peptide-1; GSP, glycated serum protein; H\&E, hematoxylin and eosin; HIG, high dose of RAGP; HOMA-IR, homeostasis model for the assessment of IR; HPLC, high-performance liquid chromatography; LOW, low dose of RAGP; LPS, lipopolysaccharide; MET, metformin; NC, normoglycemic; RAGP, purified anthraquinone glycoside preparation from rhubarb; SCFA, short-chain fatty acid; STZ, streptozotocin; T2DM, type 2 diabetes mellitus; TCM, traditional Chinese medicine; ZO-1, zonula occludens protein-1. 


\section{INTRODUCTION}

Rheum palmatum L., a perennial herb belonging to the Polygonaceae, is well-known in TCM for use in patients with constipation or gastrointestinal hemorrhage and ulcers, and it has antibacterial, anti-inflammatory properties (Zargar et al., 2011). Rhubarb contains a variety of potentially bioactive components, including anthraquinones, bianthrone, stilbenes, polysaccharides, and tannins. In many plants, anthraquinones mainly exist in a combined form (Cao and Zhou, 2009). Previous studies have shown that the total anthraquinone content of rhubarb is $\sim 4.5 \%(\mathrm{w} / \mathrm{w})$, of which free anthraquinones account for $1.9 \%(\mathrm{w} / \mathrm{w})$, while the remainder are present as glycosides (Arvindekar et al., 2015).

Type 2 diabetes mellitus (T2DM) is widespread worldwide, with an annually escalating incidence. According to statistics from the International Diabetes Federation demonstrate, there were 415 million diabetic patients worldwide in 2015, and this number will increase to 642 million by 2040 (Rocha Fernandes et al., 2016). T2DM represents a significant threat to health, but it has complex pathogenesis. T2DM involves multiple disorders, including of lipid and glucose metabolism, $\beta$-cell dysfunction, chronic low-grade inflammation, and oxidative stress, which result in insulin resistance and insufficient insulin secretion (Tangvarasittichai, 2015; Gutierrez-Rodelo et al., 2017). Rhubarb and other Chinese herbal decoctions have been used to treat diabetes and its complications (Li et al., 2004; Aditya et al., 2015), but anti-diabetic effects of anthraquinoneglycosides have rarely been reported and its mechanism are still unclear.

Recent studies have shown that gut dysbiosis is also a key underlying defect in T2DM (Han and Lin, 2014; Patterson et al., 2016). Approximatively 1-2 kg of bacteria resides in the human body, $90 \%$ of which live in the intestine (Wang, 2017). The balance of the gut microbiota is of great significance for the maintenance of appropriate whole-body metabolism (Jiang, 2014). Furthermore, there is now a large volume of literature to show that imbalance in the gut microbiome is associated with diseases, including obesity, T2DM, and liver cirrhosis (Qin et al., 2012; Karlsson et al., 2013; Qin et al., 2014). The gut microbiota and their metabolites can influence energy balance and glucose metabolism, and induce low-grade inflammation, all of which are important factors for the development of T2DM (Chassaing and Gewirtz, 2014; Mikkelsen et al., 2015). However, previous paper have reported that dietary fiber intake can increase the abundance of SCFA-producing bacteria in the intestine, which can ameliorate T2DM (Zhao L.P. et al., 2018). SCFAs are a stimulus for the secretion of glucagon-likepeptide1 (GLP-1), the action of which is a target for the treatment of T2DM. Among the TCMs that have been investigated for their effects in T2DM, xiexin tang has been shown to ameliorate T2DM in rats by modulating the gut microbiota (Wei et al., 2018). In addition, emodin has been shown to ameliorate chronic kidney disease by reducing the number of harmful bacteria and altering the gut microbial composition (Zeng et al., 2016). Anthraquinone glycosides have been shown to be mainly absorbed in the intestine and have a bacteriostatic effect as well as anti-inflammatory effects (Zargar et al., 2011; Luo et al., 2013). Yu et al. (2018) also found that the antibacterial activity of anthraquinone-glycoside against pathogenic bacteria is stronger than that of probiotics by culturing several probiotics and pathogenic bacteria in vitro. It is unclear whether the hypoglycemic mechanism of anthraquinone-glycosides is related to the regulation of gut microbiota.

Therefore, we aimed to determine whether purified anthraquinone-glycoside preparation from rhubarb (RAGP) can ameliorate T2DM by modulating the gut microbiota and/or having an anti-inflammation effect.

\section{MATERIALS AND METHODS}

\section{Materials}

The dried rhizomes of $R$. palmatum were obtained from Zhejiang Chinese Medicine University Medicine Co., Ltd. (Zhejiang, China). Diaion HP-20 macroporous resin was purchased from Mitsubishi Group (Tokyo, Japan). Emodin standard and STZ was purchased from Aladdin Bio-Reagents (Shanghai, China). Metformin was purchased from China Associate Pharmaceutical Co., Ltd. (Shenzhen, China). Glucose, GSP, insulin, and GLP-1 kits were purchased from Nanjing Jiancheng Biology Technology Co., Ltd. (Jiangsu, China). A MagMK Soil Genome DNA Extraction kit was purchased from Sangon Biotech Co., Ltd., (Shanghai, China). Antibodies against occludin, ZO- 1 and $\beta$-actin were purchased from Wanlei Biology Technology Co., Ltd. (Liaoning, China). All the reagents were of analytical or HPLC grade.

\section{Preparation of RAGP}

Three kilograms of rhubarb powder was reflux-extracted with six times the volume of $80 \%$ ethanol three times for $1.5 \mathrm{~h}$ each. The combined filtrate was then concentrated and the ethanol removed using a reduced pressure evaporator. The extractum was then mixed 1:1 in distilled water by ultrasonication. Following this, the solution was extracted with petroleum ether, ethyl acetate, and $n$-butanol, in sequence, with the $n$-butanol fraction containing the anthraquinone glycosides. This crude extract was purified by chromatography using a Diaion HP-20 column, and successively eluted with three times the column volume of distilled water, $10 \% \mathrm{MeOH}, 20 \% \mathrm{MeOH}, 40 \% \mathrm{MeOH}$, $60 \% \mathrm{MeOH}$, and $80 \% \mathrm{MeOH}$. The eluates were collected separately and analyzed by thin layer chromatography. The 40 and $60 \% \mathrm{MeOH}$ eluates were combined and dried using the reduced pressure evaporator to obtain the purified total anthraquinone glycoside extract. This extract was separated and the components identified as EMG, AEG, and CPG by physicochemical and spectroscopic analysis in our laboratory, and calibrated successively to purities of $98.56,98.13$, and $99.32 \%$ using the HPLC peak area normalization method. The total anthraquinone glycoside content was then determined by UV spectrophotometry using EMG as a standard.

The anthraquinone glycoside (EMG, AEG, and CPG) content was determined by HPLC. Chromatographic analysis was undertaken using an Agilent Extend-C18 (4.6 mm × $250 \mathrm{~mm}$, 
$5 \mu \mathrm{m}$ ) column and a mobile phase of acetonitrile: $0.1 \%$ formic acid $(90: 10, \mathrm{v} / \mathrm{v})$. The flow rate was $1.0 \mathrm{~mL} / \mathrm{min}$, the detection wavelength was $267 \mathrm{~nm}$, the column temperature was $30^{\circ} \mathrm{C}$, and $20 \mu \mathrm{L}$ of sample was used.

\section{Animal Experiments}

Male Sprague-Dawley rats (100-120 g) were acclimated to the experimental conditions of $20 \pm 2^{\circ} \mathrm{C}$, humidity $60 \pm 5 \%$, 12 h light/dark cycle, and ad libitum food and water, for 1 week. Rats and food were purchased from the Laboratory Animal Center of Zhejiang Academy of Medical Sciences (Zhejiang, China; Certificate Number SCXK 2014-0001). All animal experimentation procedures were conducted in accordance with the Chinese Guidelines for Animal Care, which conform with the internationally accepted uses of experimental animals.

T2DM was induced by consumption of a high fat-high sucrose diet, containing 20\% lard, 20\% sucrose, and $2.5 \%$ cholesterol, for 12 weeks, followed by an intraperitoneal (i.p.) injection of a single low dose of STZ dissolved in normal saline $(35 \mathrm{mg} / \mathrm{kg}$ ) ( $\mathrm{Li}$ et al., 2017). Rats in the control group received an equivalent volume of normal saline. T2DM was confirmed by retro-orbital blood sampling $48 \mathrm{~h}$ after STZ injection for the measurement of FBG. An FBG $>7.0 \mathrm{mmol} / \mathrm{L}$ was taken to indicate diabetes, and the rat was included in the study. All the included rats were randomly divided into five groups ( $n=6$ per group): normoglycemic rats administered distilled water (NC); T2DM rats administered distilled water (T2DM); T2DM rats administered metformin (MET, $100 \mathrm{mg} / \mathrm{kg})$; T2DM rats administered a LOW (100 mg/kg); and T2DM rats administered a HIG (400 mg/kg). Each rat was gavaged once daily for 6 weeks. This dose administered to T2DM rats has proven to be safe and effective by preliminary testing. During this time, all rats were allowed free access to food and water, and their body masses were recorded weekly.

\section{Biochemical Analyses}

After 6 weeks of RAGP, metformin, or water administration, the rats were terminally anesthetized using 3.5\% chloral hydrate, i.p. Blood samples were collected from the abdominal aorta and centrifuged at $3,500 \times g$ for $20 \mathrm{~min}$ at $4^{\circ} \mathrm{C}$ for serum collection. Serum FBG, GSP, insulin, and GLP-1 were measured according to the instructions of the commercially available kits, and then measured on a microplate reader (Multiskan FC, Thermo Fisher Scientific, Waltham, MA, United States). The HOMA-IR was calculated for each rat using the following equation:

HOMA-IR $=[$ FBG $(\mathrm{mmol} / \mathrm{L}) \times$ fasting insulin $(\mathrm{mIU} / \mathrm{L})] / 22.5$.

\section{Histological Examination}

Pancreas and ileum were dissected and then washed with phosphate-buffered saline. The tissues were fixed with $4 \%$ paraformaldehyde for $24 \mathrm{~h}$, embedded in paraffin, cut into 5$\mu \mathrm{m}$-thick sections, stained with $\mathrm{H} \& \mathrm{E}$, and examined using a light microscope (BX20, Olympus, Tokyo, Japan).

\section{Western Blotting}

Portions of ileum were lysed with protease inhibitor, and the protein content of each quantified using a bicinchoninic acid protein assay kit. Equal amounts of protein (50 $\mu \mathrm{g}$ per lane) were resolved on $12 \%$ polyacrylamide gels and transferred to polyvinylidene fluoride membranes (Millipore, Marlborough, MA, United States). After blocking with 4\% skim milk for $3 \mathrm{~h}$, membranes were incubated overnight with specific primary antibodies against occludin, ZO-1 or $\beta$-actin at room temperature. Each membrane was subsequently washed three times with tris-buffered saline-Tween-20 for $5 \mathrm{~min}$ and then incubated with horseradish peroxidase-conjugated secondary antibody for $2 \mathrm{~h}$ at $4^{\circ} \mathrm{C}$. The proteins were then visualized using an enhanced chemiluminescence detection system (Amersham Pharmacia, Piscataway, NJ, United States).

\section{Gut Microbiome Analysis}

The total fecal genome was extracted under aseptic conditions using a Mag-MK Soil Genome DNA Extraction kit. PCR amplification of the $\mathrm{V} 3-\mathrm{V} 4$ region of $16 \mathrm{~S}$ rDNA was then performed, and then the purified amplicons were analyzed using paired-end sequencing on the Illumina MiSeq platform by GENEWIZ Technology Inc software (Jiangsu, China), and the raw sequences were processed and analyzed using the Quantitative Insights into Microbial Ecology (QIIME; Version 1.9.1) software package. All sequences were clustered into operational taxonomic units (OTUs) according to $97 \%$ similarity, principal component analysis (PCA) was conducted, the alpha diversity index was calculated, and the relative abundance of each bacterial taxon was analyzed by comparison with Silva128 16S rRNA database and QIIME.

\section{Statistical Analysis}

Data were analyzed by one-way ANOVA using SPSS software (Version 16.0) and are expressed as mean \pm SD. Significant treatment differences were identified using Tukey's multiple comparison test. Spearman's correlation analyses were performed to identify relationships between variables. $P<0.05$ was considered to represent statistical significance.

\section{RESULTS AND DISCUSSION}

\section{Quantification of Active Ingredients in the Purified RAGP}

It is recognized that the active ingredients of rhubarb are mainly anthraquinones, which exist in free or glycosidated forms in plants (Zargar et al., 2011). The total anthraquinone glycoside content was determined to be $58 \%$ in RAGP. The content of EMG, AEG, and CPG in RAGP were $2.05,6.61$, and $3.73 \%$, respectively, as determined by HPLC (Figure 1).

\section{Effects of RAGP on Body Mass and Serum Biochemistry in T2DM Rats}

In this study, we used a high-fat, high-sugar diet and low-dose STZ i.p. injection to induce diabetes in rats that mimics the characteristics of human T2DM. T2DM rats exhibited polydipsia, polyphagia, polyuria, emaciation, and weakness by observation. To monitor the progression of T2DM and the effects of therapy, 


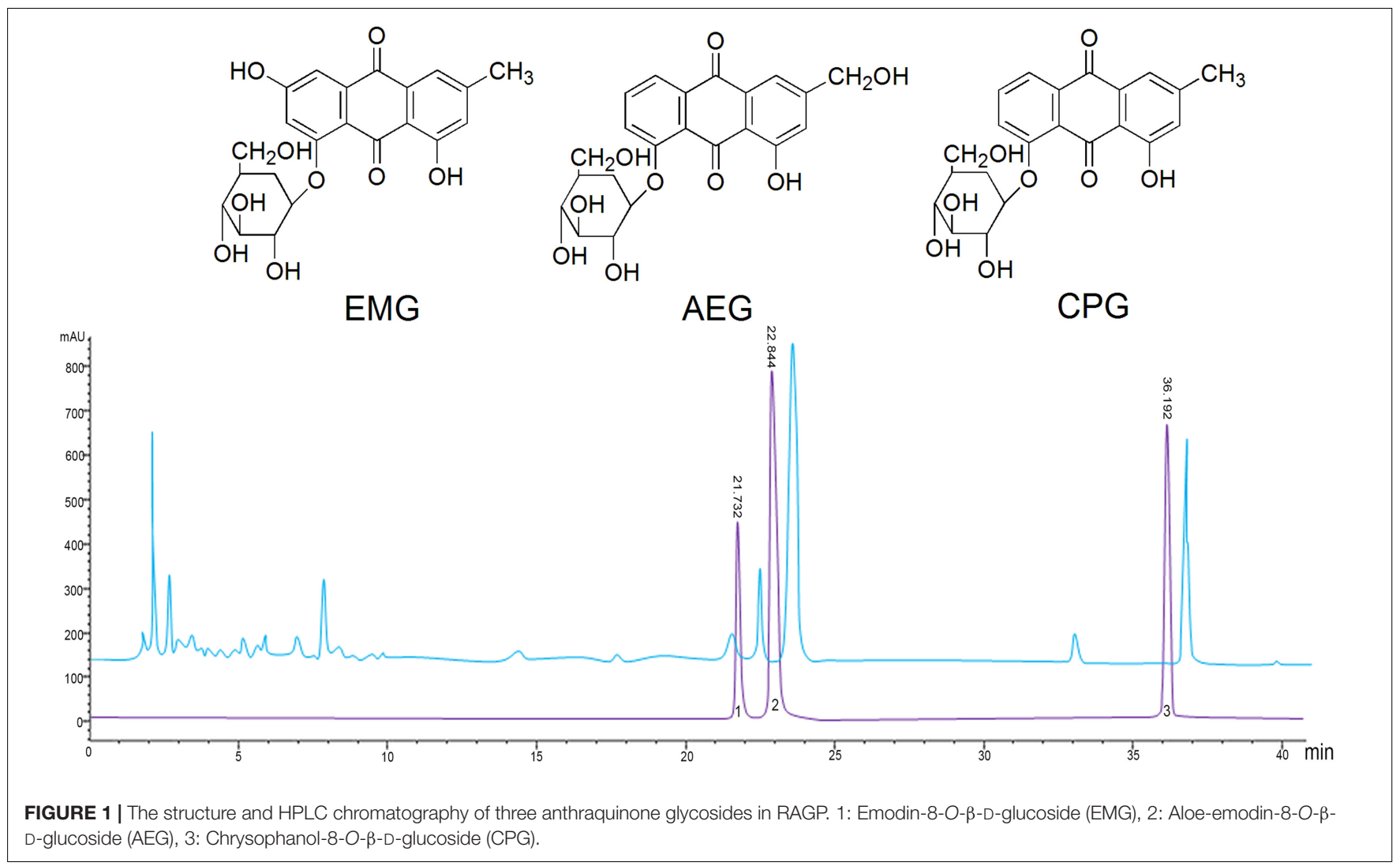

the rats were weighed weekly throughout the experiment. The rats fed high-fat, high-sugar diets for 12 weeks had higher body mass than those in NC group, but after injection of STZ (week 12 ), this decreased rapidly in T2DM rats, while continuing to increase in the NC group. From weeks 13 to 18, the rats in NC group demonstrated a normal mental state and gained body mass steadily, while rats in T2DM group demonstrated an impaired mental state and decreasing body mass $(P<0.05)$. However, the T2DM rats in MET, LOW, and HIG groups slowly regained body mass, and high-dose RAGP treatment had a greater effect (Figure 2A, $P<0.05$ ).

Abnormal glucose metabolism and insufficient insulin secretion are both features of diabetes (Azofeifa et al., 2016). Long-term high-fat diet-feeding and STZ administration causes both pancreatic $\beta$-cell damage and insulin resistance (Zhang et al., 2018). At the end of week 12, the FBG and GSP concentrations in T2DM rats were $>7.0$ and $2.2 \mathrm{mmol} / \mathrm{L}$, respectively, indicating that T2DM rats were induced successfully. The GSP concentration reflects the mean blood glucose concentration over the preceding 2-3 weeks, which is useful for the diagnosis and monitoring of diabetic patients (Chen, 2011). At the end of week 18, the FBG and GSP concentrations in untreated T2DM rats were significantly higher than those of the NC group $(P<0.01)$. However, these were much lower in the MET, LOW, and HIG groups than in the T2DM group (Figures 2B,C, $P<0.01)$. Metformin is one of the most commonly used oral hypoglycemic agents in the clinic, and its hypoglycemic mechanism is mainly through reducing liver gluconeogenesis and inhibiting the intestinal absorption of sugar (Ferrucci and Fabbri, 2018). Therefore, the hypoglycemic effect of metformin was stronger than that of RAGP, but their hypoglycemic mechanism was different.

GLP-1 is a peptide hormone coded by the proglucagon gene and secreted by intestinal epithelial L cells, the action of which is a target of a number of drugs for T2DM (Kuhre et al., 2015). GLP-1 has been shown to increase insulin sensitivity, stimulate insulin secretion, reduce islet $\beta$ cell apoptosis, promote $\beta$-cell proliferation, protect islet cells from glucose toxicity and inflammatory damage, and to act on the central nervous system (especially the hypothalamus) to induce a feeling of satiety (Guo et al., 2015). Therefore, the serum GLP-1 concentration is also important in T2DM. As shown in Figure 2D, GLP-1 concentration was significantly decreased in T2DM group compared with NC group, and metformin and RAGP significantly promoted the secretion of GLP-1 in T2DM rats $(P<0.01)$. GLP-1 binds to its receptors on islet $\beta$-cells, increasing the intracellular concentration of cAMP. The consequent activation of protein kinase A (PKA) stimulates insulin secretion and promotes $\beta$-cell growth (Purves et al., 2009; Holst et al., 2011). Serum insulin and HOMAIR was significantly higher in the T2DM group than in the NC group, but that metformin and $400 \mathrm{mg} / \mathrm{kg}$ RAGP treatment significantly decreased serum insulin and HOMA-IR (Figures 2E,F, $P<0.01$ ). The serum insulin in LOW group did not demonstrate a significant difference, compared with T2DM group. These results imply that RAGP alleviates insulin resistance 


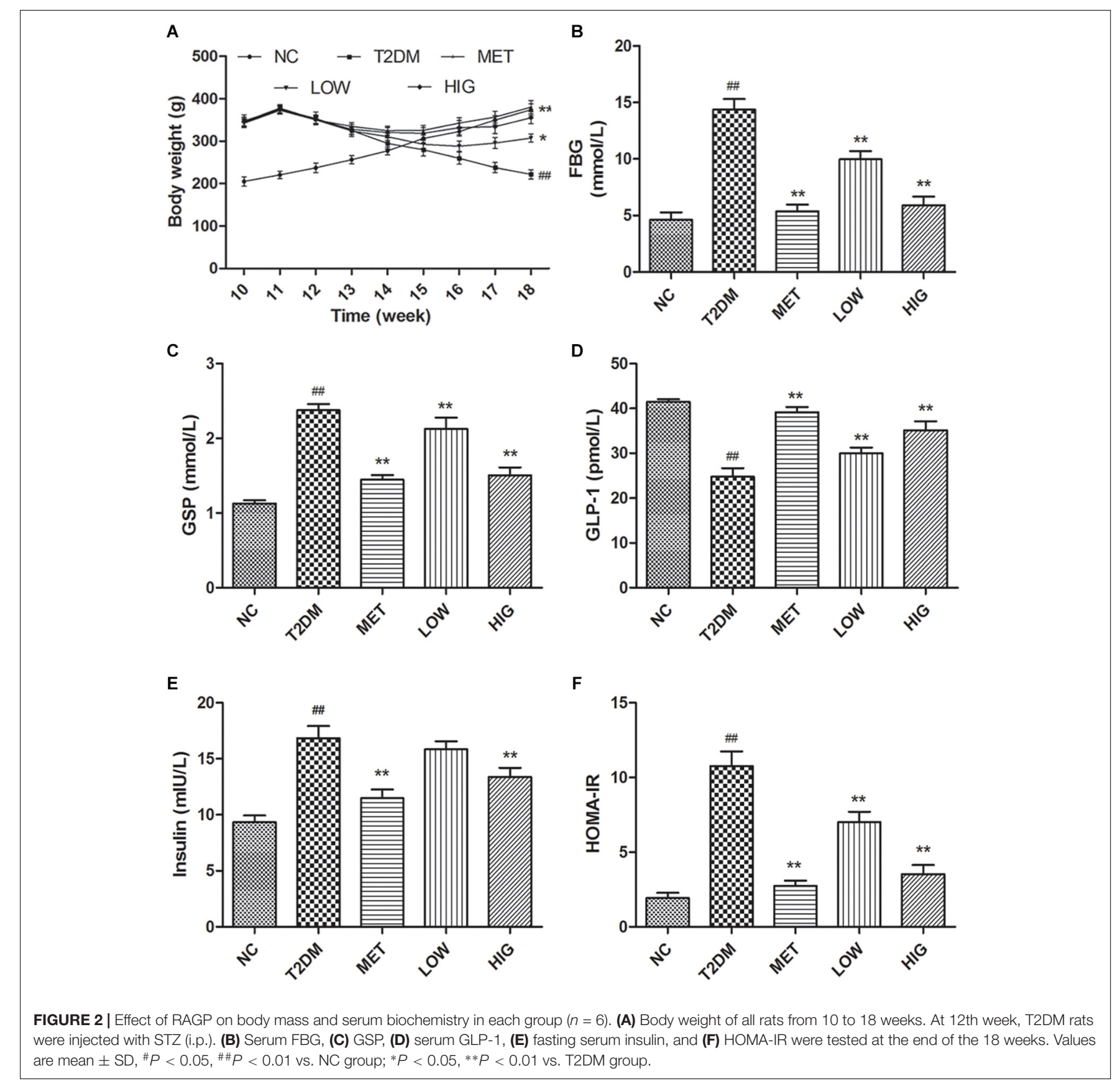

and hyperglycemia, justifying its use for the treatment of T2DM and its complications.

\section{Effects of RAGP on Pancreatic and lleum Tissue Histology}

The hyperglycemia observed in the T2DM mice is likely to be associated with the production a large quantity of superoxide, which induces pancreatic and ileum damage (Giugliano et al., 1996). Some anthraquinone compounds have been reported to have antioxidant effects (Zargar et al., 2011; Wang et al., 2016); therefore pancreatic histology was analyzed following
H\&E staining of tissue sections (Figure 3A). The islets of rats in the NC group were round or oval, with well-defined boundaries, and were filled with dense, well-distributed endocrine cells. By contrast, the islets of rats in the T2DM group were irregularly shaped, with unclear margins, nuclear pyknosis, vacuolar degeneration, inflammatory cell infiltration, and fewer $\beta$-cells. However, these features were less marked and the number of islet $\beta$-cells was significantly higher in rats in the MET and RAGP-treated groups than in the T2DM group.

Gastrointestinal dysfunction is a common complication in T2DM patients, manifesting for example as abnormal intestinal emptying, diarrhea, or constipation (Yang et al., 2015). 


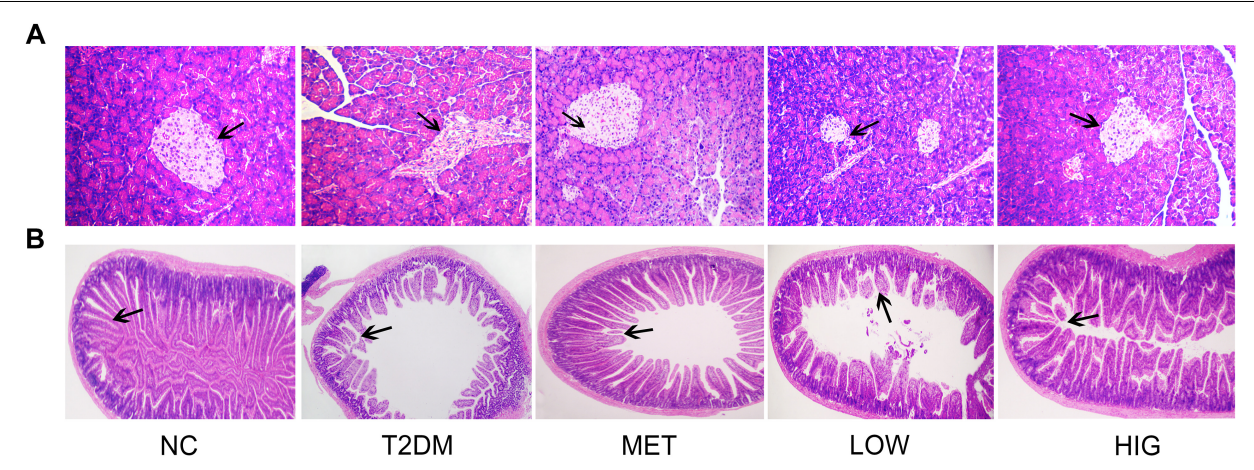

FIGURE 3 | Effects of RAGP on histopathological analysis. (A) Pancreas tissues stained with H\&E (100x); (B) ileum tissues stained with H\&E (25×).

H\&E-stained ileum sections from rats in the NC group displayed intact architecture with well-arranged villi and crypts (Figure 3B), whereas sections in T2DM group demonstrated marked abnormalities, including abnormal intestinal walls, disorganized and collapsed villi, and swollen and degenerate villus epithelium. However, MET, LOW, or HIG groups were associated with less intestinal pathology, and this effect was more pronounced in the HIG group. These findings are in agreement with those of a previous study (Zhang et al., 2017).

\section{Effects of RAGP on Intestinal Integrity}

The chronic low levels of inflammation present in the gut of diabetic patients are associated with the degradation of intestinal tight junctions (Fava, 2014). ZO-1 and occludin are major tight junction proteins found in the intestine; if these are present at lower concentrations, greater intestinal permeability results (Sato et al., 2017). Previous studies have found that the small intestinal integrity of diabetic patients is impaired because of the thinning of the intestinal mucus layer, a lack of particular antimicrobial peptides and tight junction proteins, and oxidative stress (Brun et al., 2007; Cani et al., 2009). As shown in Figure 4A, the protein expression of ZO-1 and occludin was significantly lower in T2DM group. However, treatment with metformin or high-dose RAGP significantly increased the expression of ZO-1 and occludin (Figure 4B, $P<0.01$ ), but low-dose RAGP had no obvious effect on ZO-1. Low tight junction protein expression in the intestine of diabetic patients increases intestinal permeability, such that the lipopolysaccharide (LPS) produced by Gram-negative bacteria can be absorbed in larger quantities, causing chronic inflammation by activating the NF- $\kappa$ B signaling pathway (Cani et al., 2009). It has been also shown that emodin reduces inflammation in rats with acute pancreatitis (Ma et al., 2005). Moreover, RAGP in intestine can be decomposed and inhibit the activity of $\mathrm{Na}^{+}-\mathrm{K}^{+}$-ATPase, which promotes intestinal water secretion (Xie et al., 2018). Hattori et al. (1982) and Song et al. (2011) also found that anthraquinone-glycosides entering into the intestinal tract are mainly decomposed into a glycone by enzymes ( $\beta$-glucosidase) secreted by the intestinal flora, thereby exerting antibacterial and anti-inflammatory action. Therefore, it may promote the growth of probiotics and inhibit the production of LPS, thereby upregulating the expression of tight junction proteins and increasing intestinal barrier function. The RAGP-induced improvement in intestinal integrity should therefore reduce this LPS-induced inflammation (Figure 7). Our findings are consistent with those of a previous study that found that RAGP can inhibit inflammatory responses by protecting intestinal tight junctions (Zheng et al., 2018).

\section{Effects of RAGP on Gut Microbiome Composition in T2DM Rats}

The secretion of GLP-1 closely correlates with the quantity of SCFA produced by gut microbial metabolism (Everard and Cani, 2013; Park et al., 2015). Increasing the abundance of SCFA-producing bacteria is another potential approach to the treatment of T2DM. To explore the effects of RAGP on the gut microbiome, Illumina MiSeq sequencing of the V3-V4 region of $16 \mathrm{~S}$ rDNA was performed. A total of 1,981,228 raw sequences were generated, from which low-quality sequences were removed, leaving $88.1 \%$ of the total. The remaining clean tags were clustered into OTUs on the basis of $97 \%$ similarity.

The human body carries a huge and varied microbiota, in total $\sim 10$ times the number of human cells (Zhang, 2017). Diversity of its microbiota is thought to indicate a "healthy gut" (Sommer et al., 2017). $\alpha$-Diversity analysis of the intestinal contents showed that the Chao 1 and Shannon values for the T2DM group were significantly lower than those for the NC group, indicating that the abundance and diversity of the gut microbiome in T2DM rats was lower $(P<0.01)$, but these values were higher in MET and HIG groups than in T2DM group (Figures 5A,B, $P<0.05$ ). These results are consistent with those of Zhang et al. (2018). Greater microbial diversity is associated with a more robust response to environmental insults, because functionally similar microbes in an intact ecosystem can compensate for the function of other missing species (Sommer et al., 2017). To analyze the $\beta$-diversity of the gut microbiome among the rat groups, PCA was conducted, which clusters the gut microbial taxa for each group (Figure 5C).

At the phylum level, the gut microbiome consists largely of Firmicutes, Bacteroidetes, Proteobacteria, Actinobacteria, and Verrucomicrobia, of which the Firmicutes and Bacteroidetes 

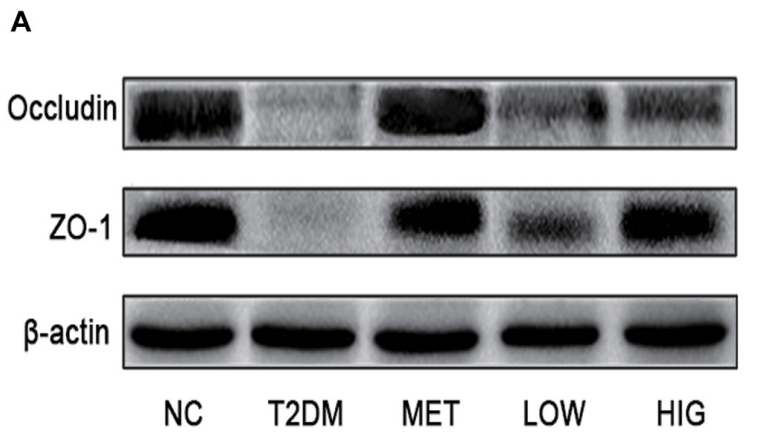

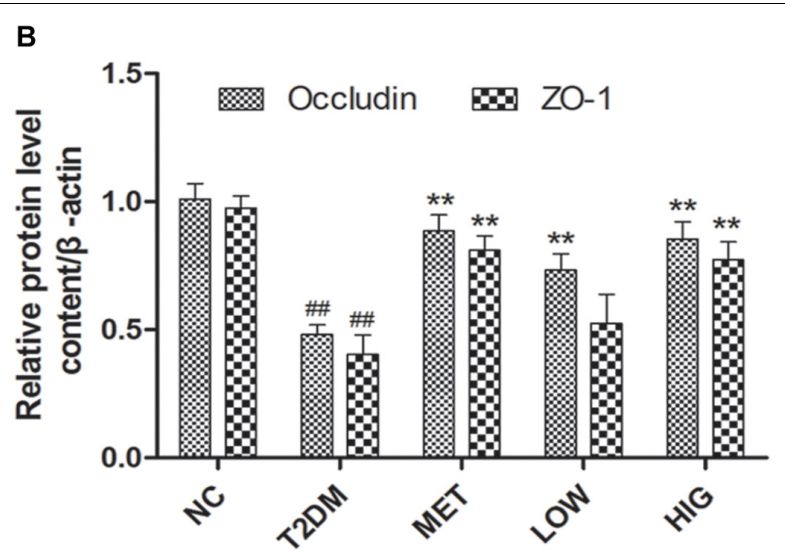

FIGURE 4 | Effects of RAGP on the expression level of ZO-1 and occludin in the ileum tissues in each group ( $n=6)$. (A) The expression levels of ZO-1 and occludin were analyzed by western blotting. (B) The density identification of occluding, ZO-1 were calculated as shown. Values are mean \pm SD, \# $P<0.05$, \#\# $P<0.01$ vs. NC group; ${ }^{*} P<0.05,{ }^{* *} P<0.01$ vs. T2DM group.

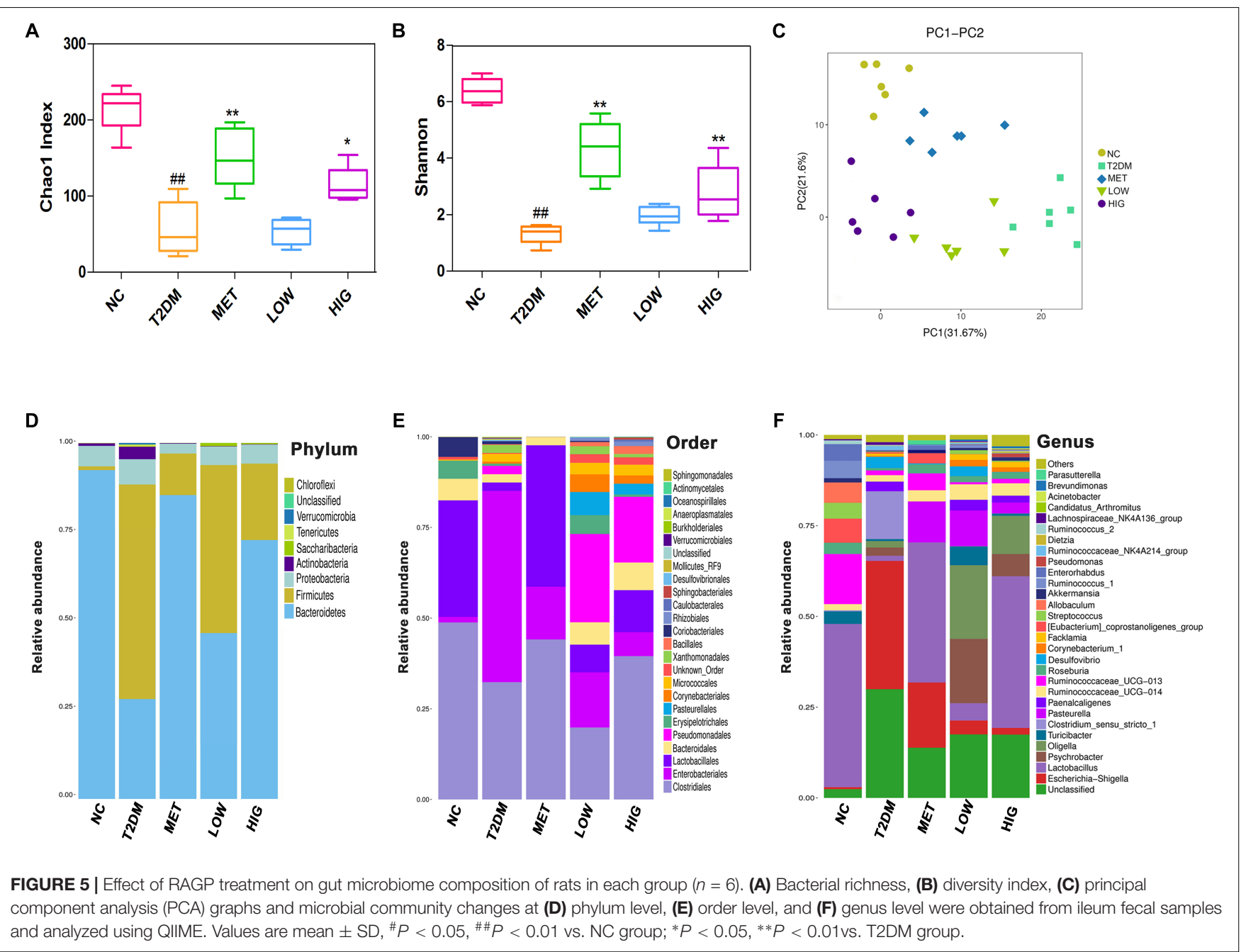

account for $98 \%$ of the total number (Wu et al., 2010). Mahowald et al. (2009) found that Bacteroidetes is the only phylum that encodes carbohydrate-degrading enzymes in the human gut microbiome. Frank et al. (2014) transplanted feces from obese rats with a high proportion of Firmicutes and Bacteroidetes into sterile mice, which successfully replicated the obese 
phenotype. This implies that a change in the abundance of Firmicutes and Bacteroidetes can induce the development of obesity and diabetes. In our study, the gut microbiome of the rats was mainly composed of Bacteroidetes and Firmicutes, and greater abundance of Firmicutes and lower abundance of Bacteroidetes characterized the T2DM group when compared with the NC group, while metformin and RAGP treatment significantly reduced these differences (Figure 5D). Rhein inhibits the activity of Firmicutes and promotes the degradation of carbohydrates by Bacteroidetes (Wang et al., 2016). Zhao C. et al. (2018) also found that the abundance of Bacteroidetes is significantly lower in diabetic animals, but is increased by treatment with an extract of the brown seaweed, Lessonia nigrescens.

At the taxonomic order level, the abundance of Clostridiales and Lactobacillales was greater in the MET and HIG groups than in the T2DM group, whereas the abundance of Enterobacteriales was lower in the MET and HIG groups (Figure 5E).

At the genus level, some probiotic and SCFA-producing bacteria are associated with diabetes resistance (Patterson et al., 2016). SCFA-producing bacteria can metabolize polysaccharides, such as insoluble starch and indigestible fructose, into SCFAs, including acetate, propionate, and butyrate. These SCFAs act as energy substrates and bind to G-protein-coupled receptor 43/41(GPCR 43/41), causing secretion of additional GLP-1, which ameliorates insulin resistance and lowers blood glucose (Holst et al., 2011; Park et al., 2015). As shown in Figures 5F, 6, significantly fewer Lactobacillus, Roseburia, and Akkermansia were present in T2DM rats, but their numbers were significantly higher in the MET and HIG than those in the T2DM group $(P<0.01)$. In contrast, the abundances of Desulfovibrio and Lachnospiraceae NK4A136 were much higher in T2DM rats
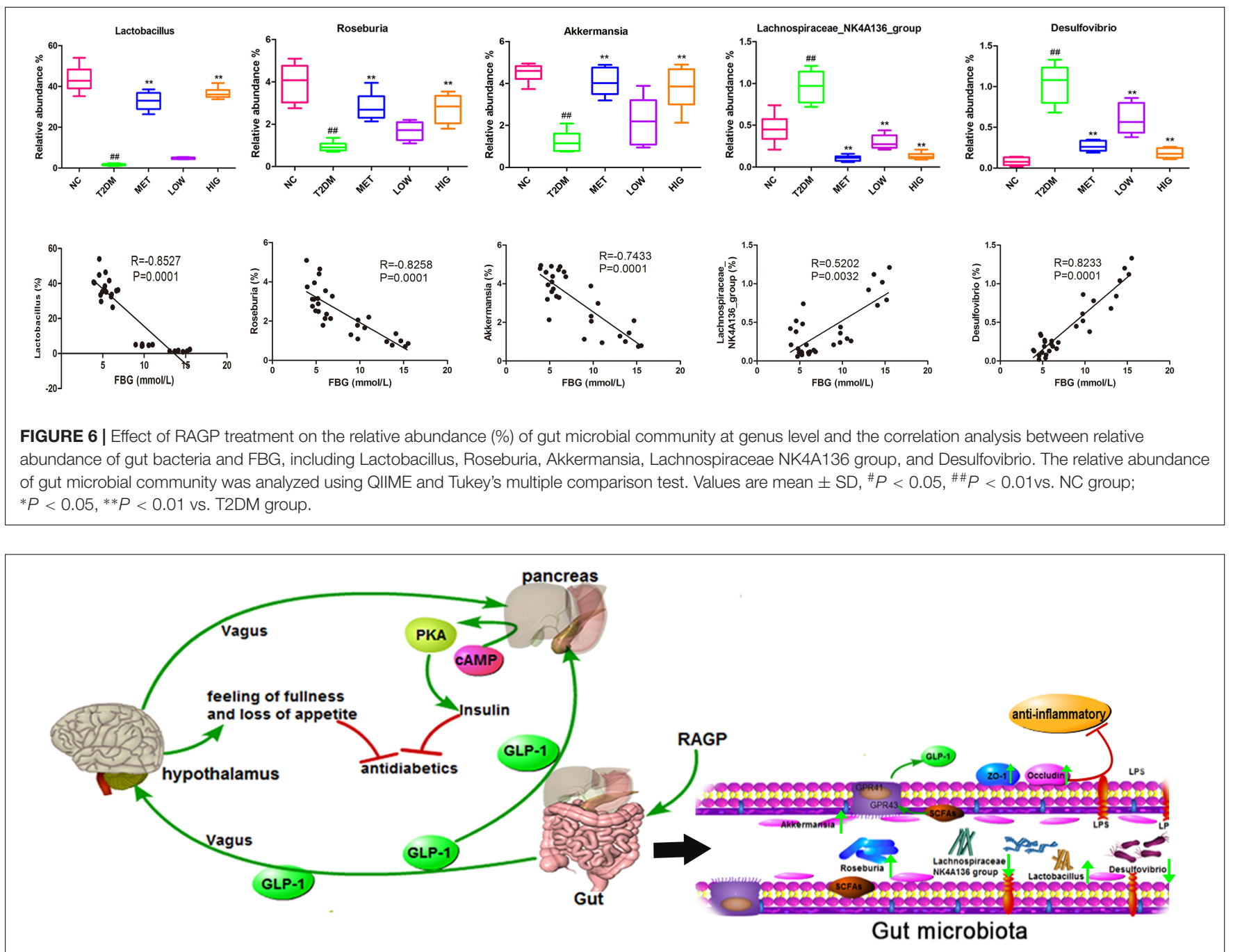

FIGURE 7 | Mechanism of RAGP against T2DM: the interaction of gut microbiota and the reduction of inflammation. RAGP regulated the structure of the gut microbiota, increased the abundance of Lactobacillus and SCFAs-producing (Roseburia and Akkermansia), and decreased Lachnospiraceae NK4A136 group and LPS-producing (Desulfovibrio). Meanwhile, RAGP up-regulated the expression level of ZO-1 and occludin, which improved intestinal integrity and suppressed the LPS-induced inflammation. The improved effect was also indicated by the green arrow after RAGP treatment. 
than in NC rats, but their abundances were markedly decreased in MET and RAGP-treated groups compared with T2DM group $(P<0.01)$. The results indicate that RAGP has a regulatory effect on the growth of probiotics and pathogenic bacteria in the intestine. Lactobacillus, a major probiotic, produces lactic acid, $\mathrm{CO}_{2}$, acetic acid, and/or ethanol, which contribute to a more acidic environment through homo- or heterofermentative metabolism (Macfarlane and Macfarlane, 1997; Nobaek et al., 2000). This is a beneficial property that makes this genus for the health of the intestinal tract. Our results were consistent with those of previous studies in which it was shown that Lactobacillus abundance was lower in diabetic and obese rats but greater in diabetic rats that were under treatment (Yan et al., 2016; Zhang et al., 2018). Roseburia is a butyric acid-producing genus that plays a key role in regulating glycolipid metabolism (Udayappan et al., 2014). Butyrate has been shown to induce the secretion of GLP-1, which in turn stimulates insulin secretion (Everard and Cani, 2013). Qin et al. (2012) found that the gut microbiome is disordered in T2DM patients, with a smaller population of certain butyrate-producing bacteria and larger numbers of opportunistic pathogens. Akkermansia is a dominant bacterial genus that colonizes the intestinal mucosa, degrades mucin, and produces propionic acid, which provides energy for intestinal epithelial cells, thereby protecting the intestinal mucosal barrier and reducing protein deposition (Collado et al., 2007). Under normal conditions, Akkermansia accounts for 3-5\% of the gut microbiome and it also protects the intestinal mucosal barrier by reducing the inflammatory response and improving glycolipid metabolism. However, in high-fat diet-fed rodents and diabetic patients, its abundance is lower, whereas it has also been shown that increasing the abundance of Akkermansia can ameliorated T2DM (Abbeele et al., 2011; He et al., 2016). The Lachnospiraceae NK4A136 group is an indicator of gut dysbiosis, because when present in great abundance, gut dysbiosis is more severe (Zheng et al., 2018).

Gut dysbiosis in T2DM patients usually involves greater abundance of Gram-negative bacteria. LPS is a component of the outer membrane of Gram-negative bacteria, and their death causes the release of a large quantity of LPS, which can be absorbed into the bloodstream and cause chronic inflammation and insulin resistance (Wang et al., 2018; Wei et al., 2018). Desulfovibrio is a Gram-negative genus that can convert sulfate into a toxic gas $\left(\mathrm{H}_{2} \mathrm{~S}\right)$, which when produced in high concentrations leads to the release of a large amount of LPS, which can destroy the intestinal barrier and increase intestinal permeability (Xiao et al., 2014). Thus, our data suggest that RAGP may ameliorate the disturbance in glucose metabolism by correcting gut microbial imbalance in T2DM rats.

\section{Correlations Between Metabolic Biomarkers and Bacterial Abundance}

To elucidate the relationships between bacterial abundance and FBG, Spearman's correlation coefficients were calculated. The abundances of Lactobacillus, Roseburia, and Akkermansia were negatively correlated with FBG, while those of Desulfovibrio and the Lachnospiraceae NK4A136 group were positively correlated (Figure 6, $P<0.01$ ), which is in agreement with previously published findings (Zhang et al., 2018; Zheng et al., 2018).

\section{CONCLUSION}

This study demonstrated that RAGP significantly ameliorates T2DM induced by high-fat diet-feeding combined with STZ administration. The mechanism can be attributed to the moderating effect on the gut microbiota. RAGP increased the abundance of certain probiotic and SCFA-producing bacteria, which provide energy for intestinal L cells to secrete more GLP-1. This GLP- 1 can then bind to receptors on islet $\beta$-cells and increase insulin secretion, as well as improving the sensitivity of peripheral tissues to insulin, thereby ameliorating insulin resistance. GLP-1 also stimulates the satiety center of the hypothalamic to reduce appetite (Figure 7). In addition, RAGP reduced the abundance of LPS-producing bacteria and enhanced intestinal integrity, which would inhibit chronic systemic inflammatory responses. Thus, our findings support the use of RAGP for the treatment of T2DM as a novel approach targeting the correction of gut dysbiosis.

\section{DATA AVAILABILITY}

All datasets generated for this study are included in the manuscript and/or the Supplementary Files.

\section{ETHICS STATEMENT}

Rats and their feed were purchased from the Laboratory Animal Center of Zhejiang Academy of Medical Sciences (Zhejiang, China; Certificate Number SCXK 2014-0001). All animal experimentation procedures were conducted in accordance with the Chinese Guidelines for Animal Care, which conform with the internationally accepted uses of experimental animals.

\section{AUTHOR CONTRIBUTIONS}

H-XC, L-SZ, YL, and KY designed the study. H-XC, L-SZ, YL, $\mathrm{Z}-\mathrm{YH}, \mathrm{KY}$, and YG conducted the experiments and discussed the results. $\mathrm{H}-\mathrm{XC}, \mathrm{L}-\mathrm{SZ}$, and $\mathrm{KY}$ wrote the manuscript.

\section{FUNDING}

This project was supported by the Zhejiang Provincial Science Foundation of China (Grant No. LY16H280007).

\section{SUPPLEMENTARY MATERIAL}

The Supplementary Material for this article can be found online at: https://www.frontiersin.org/articles/10.3389/fmicb.2019. 01423/full\#supplementary-material 


\section{REFERENCES}

Abbeele, P. V., Gérard, P., Rabot, S., Bruneau, A., El Aidy, S., Derrien, M., et al. (2011). Arabinoxylans and inulin differentially modulate the mucosal and luminal gut microbiota and mucindegradation in humanized rats. Environ. Microbiol. 13, 2667-2680. doi: 10.1111/j.1462-2920.2011.02533.x

Aditya, A., Tanaji, M., Panan, P., Kirti, L., Nanda, G., and Akalpita, A. (2015). Evaluation of anti-diabetic and alpha glucosidase inhibitory action of anthraquinones from Rheum emodi. Food Funct. 6, 2693-2700. doi: 10.1039/ c5fo00519a

Arvindekar, A. U., Pereira, G. R., and Laddha, K. S. (2015). Assessment of conventional and novel extraction techniques on extraction efficiency of five anthraquinones from Rheum emodi. J. Food Sci. Technol. 52, 6574-6582. doi: 10.1007/s13197-015-1814-3

Azofeifa, G., Quesada, S., Navarro, L., Hidalgo, O., Portet, K., Perez, A. M., et al. (2016). Hypoglycaemic, hypolipidaemic and antioxidant effects of blackberry beverage consumption in streptozotocin-induced diabetic rats. J. Funct. Foods $26,330-337$

Brun, P., Castagliuolo, L., Leo, V. D., Buda, A., Pinzani, M., Palù, G., et al. (2007). Increased intestinal permeability in obese mice: new evidence in the pathogenesis of nonalcoholic steatohepatitis. Am. J. Physiol. Gastrointest. Liver Physiol. 292, 518-525.

Cani, P. D., Possemiers, S., Van de Wiele, T., Guiot, Y., Everard, A., Rottier, O., et al. (2009). Changes in gut microbiota control inflammation in obese mice through a mechanism involving GLP-2-driven improvement of gut permeability. Gut 58, 1091-1103. doi: $10.1136 /$ gut.2008.165886

Cao, L., and Zhou, J. J. (2009). Research progress in anthraquinones. J. Pharm. Res. $24,237-238$.

Chassaing, B., and Gewirtz, A. T. (2014). Gut microbiota, low-grade inflammation, and metabolic syndrome. Toxicol. Pathol. 42, 49-53. doi: $10.1177 / 0192623313508481$

Chen, S. J. (2011). The clinical significan of combined determination fasting blood sugar and saccharifying serum protein in the diagnosis of diabetes. Chin. Prac Med. 6, 28-29.

Collado, M. C., Derrien, M., Isolauri, E., de Vos, W. M., and Salminen, S. (2007). Intestinal integrity and Akkermansia muciniphila, a mucindegrading member of the intestinal microbiota present in infants, adults, and the elderly. Appl. Environ. Microbiol. 73, 7767-7770.

Everard, A., and Cani, P. D. (2013). Diabetes, obesity and gut microbiota. Best Pract. Res. Clin. Gastroenterol. 27, 73-83. doi: 10.1016/j.bpg.2013.03.007

Fava, S. (2014). Glucagon-like peptide 1 and the cardiovascular system. Curr. Diabetes Rev. 10, 302-310.

Ferrucci, L., and Fabbri, E. (2018). Inflammageing: chronic inflammation in ageing, cardiovascular disease, and frailty. Nat. Rev. Cardiol. 15, 505-522. doi: 10.1038/ s41569-018-0064-2

Frank, A. D., Yassine, S., Patricia, L., Fabienne, D., Bénédicte, L., Joël, D., et al. (2014). Replication of obesity and associated signaling pathways through transfer of microbiota from obese prone rat. Diabetes 63, 1624-1636.

Giugliano, D., Ceriello, A., and Paolisso, G. (1996). Oxidative stress and diabetic vascular complications. Diabetes Care 19, 257-267.

Guo, S., Huang, L. M., and Xu, Y. Y. (2015). Molecular mechanism of GLP1 synthesis and secretion and its influencing factors. Prog. Physiol. Sci. 46, $115-120$.

Gutierrez-Rodelo, C., Roura-Guiberna, A., and Olivares-Reyes, J. A. (2017). Molecular mechanisms of insulin resistance: an update. Gac. Med. Mex. 153, 214-228.

Han, J. L., and Lin, H. L. (2014). Intestinal microbiota and type 2 diabetes: from mechanism insights to therapeutic perspective. World J. Gastroenterol. 20, 17737-17745. doi: 10.3748/wig.v20.i47.17737

Hattori, M., Kim, G., Motoike, S., Kobashi, K., and Namba, T. (1982). Metabolism of sennosides by intestinal flora. Chem. Pharm. Bull. 30, 1338-1346. doi: 10. 1248/cpb. 30.1338

He, K., Hu, Y., Ma, H., Zou, Z., Xiao, Y., Yang, Y., et al. (2016). Rhizoma coptidis alkaloids alleviate hyperlipidemia in B6 mice by modulating gut microbiota and bile acid pathways. Biochim. Biophys. Acta.Mole Basis Dis. 1862, 1696-1709. doi: 10.1016/j.bbadis.2016.06.006

Holst, J., Christensen, M., Lund, A., De Heer, J., Svendsen, B., Kielgast, U., et al. (2011). Regulation of glucagon secretion by incretins. Diabetes Obes. Metab. 13(Suppl. 1), 89-94. doi: 10.1111/j.1463-1326.2011.01452.x
Jiang, Y. Y. (2014). The ecological etiology analysis of type 2 diabetes rehabilitation mechanisms from gut microbiota. Med. Recapitulate 20, 3757-3759.

Karlsson, F., Tremaroli, V., Nielsen, J. J., and Backhed, F. (2013). Assessing the human gut microbiota in metabolic diseases. Diabetes Metab. Res. Rev. 62, 3341-3349.

Kuhre, R. E., Frost, C. R., Svendsen, B., and Holst, J. J. (2015). Molecular mechanisms of glucose-stimulated GLP-1 secretion from perfused rat small intestine. Diabetes Metab. Res. Rev. 64, 370-382.

Li, J. W., Yuan, K., Shang, C. S., and Guo, Y. (2017). A safer hypoglycemic agent for type 2 diabetes-berberine organic acid salt. J. Funct. Foods 38, 399-408. doi: 10.3390/molecules 24010103

Li, W. L., Zheng, H. C., Bukurub, J., and Kimpe, N. D. (2004). Natural medicines used in the traditional chinese medical system for therapy of diabetes mellitus. J. Ethnopharmacol. 92, 1-21.

Luo, P., Xu, X. Z., and Tan, Z. H. (2013). Study on the mechanisms of diarrhea effects of anthraquinone from Rheum palmatum. Pharm. Clin. Chin. Mater. Med. 29, 88-90.

Ma, W., Zhang, L. Q., and Zhang, S. H. (2005). Protective effect of emodin on lung injury in rats with severe acute pancreatitis. J. Guangdong Med. Coll. 23, 504-509. doi: 10.1080/01902140902718197

Macfarlane, G. T., and Macfarlane, S. (1997). Human colonic microbiota: ecology, physiology and metabolic potential of intestinal bacteria. Scand. J. Gastroenterol. Suppl. 222, 3-9. doi: 10.1080/00365521.1997.11720708

Mahowald, M. A., Rey, F. E., Seedorf, H., Turnbaugh, P. J., Fulton, R. S., Wollam, A., et al. (2009). Characterizing a model human gut microbiota composed of members of its two dominant bacterial phyla. Proc. Natl. Acad. Sci. U.S.A. 106, 5859-5864. doi: 10.1073/pnas.0901529106

Mikkelsen, K. H., Frost, M., Bahl, M. I., Licht, T. R., Jensen, U. S., Rosenberg, J., et al. (2015). Effect of antibiotics on gut microbiota, gut hormones and glucose metabolism. PLoS One 10:e0142352. doi: 10.1371/journal.pone.0142352

Nobaek, S., Johansson, M. L., Molin, G., Ahrne, S., and Jeppsson, B. (2000). Alteration of intestinal microflora is associated with reduction in abdominal bloating and pain in patients with irritable bowel syndrome. Am. J. Gastroenterol. 95, 1231-1238.

Park, J., Kim, M., Kang, S. G., Jannasch, A. H., Cooper, B., Patterson, J., et al. (2015). Short-chain fatty acids induce both effector and regulatory T cells by suppression of histone deacetylases and regulation of the mTOR-S6K pathway. Mucosal Immunol. 8, 80-93. doi: 10.1038/mi.2014.44

Patterson, E., Ryan, P. M., Cryan, J. F., Dinan, T. G., Ross, R. P., Fitzgerald, G. F., et al. (2016). Gut microbiota, obesity and diabetes. Postgrad. Med. J. 92, 286-300. doi: 10.1136/postgradmedj-2015-133285

Purves, G. I., Kamishima, T., Davies, L. M., Quayle, J. M., and Dart, C. (2009). Exchange protein activated by cAMP (Epac) mediates cAMP-dependent but protein kinase Ainsensitive modulation of vascular ATP-sensitive potassium channels. J. Physiol. 587, 3639-3650. doi: 10.1113/jphysiol.2009.173534

Qin, J., Li, Y., Cai, Z., Li, S., Zhu, J., Zhang, F., et al. (2012). A metagenomewide association study of gut microbiota in type 2 diabetes. Nature 490, 55-60. doi: 10.1038/nature11450

Qin, N., Yang, F., Li, A., Prifti, E., Chen, Y., Shao, L., et al. (2014). Alterations of the human gut microbiome in liver cirrhosis. Nature 513, 59-64. doi: 10.1038/ nature 13568

Rocha Fernandes, D., Ogurtsova, J., Linnenkamp, K., Guariguata, U., Seuring, L., Zhang, T., et al. (2016). IDF diabetes atlas estimates of 2014 global health expenditures on diabetes. Diabetes Res. Clin. Pract. 117, 48-54. doi: 10.1016/j. diabres.2016.04.016

Sato, J., Kanazawa, A., and Watada, H. (2017). Type 2 diabetes and bacteremia. Ann. Nutr. Metab. 71(Suppl. 1), 17-22. doi: 10.1159/000479919

Sommer, F., Anderson, J. M., Bharti, R., Raes, J., and Rosenstiel, P. (2017). The resilience of the intestinal microbiota influences health and disease. Nat. Rev. Microbiol. 15, 630-638. doi: 10.1038/nrmicro.2017.58

Song, R., Xu, L., Xu, F., Dong, H., Tian, Y., and Zhang, Z. (2011). Metabolic analysis of rhubarb extract by rat intestinal bacteria using liquid chromatographytandem mass spectrometry. Biomed. Chromatogr. 25, 417-426. doi: 10.1002/ bmc. 1467

Tangvarasittichai, S. (2015). Oxidative stress, insulin resistance, dyslipidemia and type 2 diabetes mellitus. World J. Diabetes 6, 456-480. doi: 10.4239/wjd.v6. i3.456

Udayappan, S. D., Hartstra, A. V., Dallinga, T. M., and Nieuwdorp, M. (2014). Intestinal microbiota and faecal transplantation as treatment modality for 
insulin resistance and type 2 diabetes mellitus. Clin. Exp. Immunol. 177, 24-29. doi: $10.1111 /$ cei. 12293

Wang, M. J. (2017). Research progress on intestinal flora imbalance induced type 2 diabetes. Diabetes New World 2, 197-198. doi: 10.16658/j.cnki.1672-4062.2017. 04.197

Wang, R. F., Lei, H. Y., Zang, P., and Du, H. (2016). The effect of rhein on the gut microbiota in diabetes mice. Chin. J. Microecol. 28, 21-24.

Wang, Y. L., Zhang, J., Su, F., and Ni, J. (2018). Research progress on the relationship of intestinal flora and Type 2 diabetes mellitus pathogenesis. Prac. J. Med. Pharm. 35, 77-80. doi: 10.1038/nrneph.2017.16

Wei, X. Y., Tao, J. H., Xiao, S. W., Jiang, S., Shang, E. X., Zhu, Z. H., et al. (2018). Xiexin tang improves the symptom of type 2 diabetic rats by modulation of the gut microbiota. Sci. Rep. 8:3685. doi: 10.1038/s41598-018-22094-2

Wu, X., Ma, C., Han, L., Nawaz, M., Gao, F., Zhang, X., et al. (2010). Molecular characterisation of the faecal microbiota in patients with type II diabetes. Curr. Microbiol. 61, 69-78. doi: 10.1007/s00284-010-9582-9

Xiao, S., Fei, N., Pang, X., Shen, J., Wang, L., Zhang, B., et al. (2014). A gut microbiota-targeted dietary intervention for amelioration of chronic inflammation underlying metabolic syndrome. FEMS Microbiol. Ecol. 87, 357367. doi: 10.1111/1574-6941.12228

Xie, G. Z., Hui, H. Y., Peng, M. J., and Tan, Z. J. (2018). Biotransformation of glycosides in herbal medicine by gut microbiota. Shijie Huaren Xiaohua Zazhi 26, 221-227.

Yan, X., Feng, B., Li, P., Tang, Z., and Wang, L. (2016). Microflora disturbance during progression of glucose intolerance and effect of sitagliptin: an animal study. J. Diabetes Res. 2016:2093171. doi: 10.1155/2016/2093171

Yang, Y. S., Rong, H. Y., Zhang, W. Y., Zhou, W., Chen, K., and Wang, H. (2015). Morphology observation on villi of small intestine of patients with type 2 diabetes mellitus by capsule endoscopy. Chin. J. Endoscopy 8, 798-801.

Yu, X. H., Zhang, X. R., Zhu, Y. Q., Lu, G. G., Liu, X., Dawa, L. M., et al. (2018). Study on the extraction technology and vitro antibacterial activity of rhubarb anthraquinone compounds. CJTCMP 33, 5186-5189.

Zargar, B. A., Masoodi, M. H., Ahmed, B., and Ganie, S. A. (2011). Phytoconstituents and therapeutic uses of Rheum emodi wallex Meissn. Food Chem. 128, 585-589.
Zeng, Y. Q., Dai, Z., Lu, F., Lu, Z., Liu, X., Chen, C., et al. (2016). Emodin via colonic irrigation modulates gut microbiota and reduces uremic toxins in rats with chronic kidney disease. Oncotarget 7, 17468-17478. doi: 10.18632/oncotarget. 8160

Zhang, D. (2017). Relationship between type 2 diabetes and intestinal flora. World Latest Med. Inform. 17, 252-253.

Zhang, Q., Yu, H. Y., Xiao, X., Hu, H. L., Xin, F. J., and Yu, X. B. (2018). Inulin-type fructan improves diabetic phenotype and gut microbiota profiles in rats. Peer J 6:e4446. doi: 10.7717/peerj.4446

Zhang, W. Y., Zhang, H. H., Yu, C. H., Fang, J., and Ying, H. Z. (2017). Ethanol extract of Atractylodis macrocephalae rhizoma ameliorates insulin resistance and gut microbiota in type 2 diabetic $\mathrm{db} / \mathrm{db}$ mice. J. Funct. Foods 39, 139-151.

Zhao, C., Yang, C., Chen, M., Lv, X., Liu, B., Yi, L., et al. (2018). Regulatory efficacy of brown seaweed lessonia nigrescens extract on the gene expression profile and intestinal microflora in Type 2 diabetic mice. Mol. Nutri. Food Res. 62:1700730. doi: 10.1002/mnfr.201700730

Zhao, L. P., Zhang, F., Ding, X. Y., Wu, G. J., Lan, Y. Y., Wang, X. J., et al. (2018). Gut bacteria selectively promoted by dietary fibers alleviate type 2 diabetes. Science 359, 1151-1156. doi: 10.1126/science.aao5774

Zheng, J. P., Yuan, X. B., Cheng, G., Jiao, S. M., Feng, C., Zhao, X. M., et al. (2018). Chitosan oligosaccharides improve the disturbance in glucose metabolism and reverse the dysbiosis of gut microbiota in diabetic mice. Carbohydr. Polym. 190, 77-86. doi: 10.1016/j.carbpol.2018.02.058

Conflict of Interest Statement: The authors declare that the research was conducted in the absence of any commercial or financial relationships that could be construed as a potential conflict of interest.

Copyright (c) 2019 Cui, Zhang, Luo, Yuan, Huang and Guo. This is an open-access article distributed under the terms of the Creative Commons Attribution License (CC BY). The use, distribution or reproduction in other forums is permitted, provided the original author(s) and the copyright owner(s) are credited and that the original publication in this journal is cited, in accordance with accepted academic practice. No use, distribution or reproduction is permitted which does not comply with these terms. 